\title{
Smartphone-based multimodal tethered capsule endoscopic platform for white-light, narrow-band, and fluorescence/ autofluorescence imaging
}

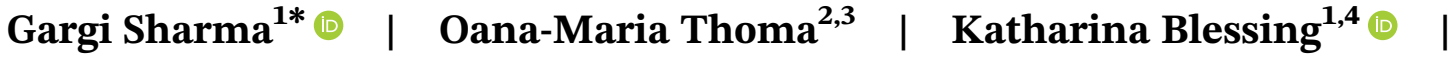 \\ Robert Gal $^{1}$ | Maximilian Waldner, ${ }^{2,3}$ | Kanwarpal Singh ${ }^{1,4}$
}

\author{
${ }^{1}$ Research Group Singh, Max-Planck \\ Institute for the Physics of Light, \\ Erlangen, Germany \\ ${ }^{2}$ Department of Medicine 1, Friedrich- \\ Alexander-Universität Erlangen- \\ Nürnberg, Erlangen, Germany \\ ${ }^{3}$ Erlangen Graduate School of Advanced \\ Optical Technologies (SAOT), Friedrich- \\ Alexander University Erlangen-Nürnberg, \\ Erlangen, Germany \\ ${ }^{4}$ Department of Physics, Friedrich- \\ Alexander Universität Erlangen- \\ Nürnberg, Erlangen, Germany

\section{*Correspondence} \\ Gargi Sharma, Max PlanckInstitute for the \\ Science of Light, Staudtstraße 2, 91058 \\ Erlangen, Germany. \\ Email: gargi.sharma@mpl.mpg.de
}

\begin{abstract}
Multimodal low-cost endoscopy is highly desirable in poor resource settings such as in developing nations. In this work, we developed a smartphone-based low-cost, reusable tethered capsule endoscopic platform that allows white-light, narrowband, and fluorescence/autofluorescence imag-

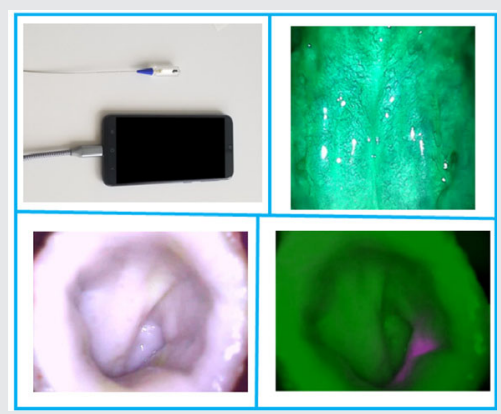
ing of the esophagus. The ex-vivo studies of swine esophagus were performed and compared with a commercial endoscope to test the white-light imaging capabilities of the endoscope. The efficacy of the capsule for narrow-band imaging was tested by imaging the vascularization of the tongue. To determine the autofluorescence/fluorescence capability of the endoscope, fluorescein dye with different concentrations was imaged. Furthermore, swine esophagus injected with fluorescein dye was imaged using the fluorescence/autofluorescence and the white-light imaging modules, exvivo. The overall cost of the capsules is approximately $12 €, 15 €$, and $42 €$ for the white light imaging, the narrow-band imaging, and the fluorescence/ autofluorescence imaging respectively. In addition, the cost of the laser source module required for the narrow-band imaging and the fluorescence/ autofluorescence imaging is approximately $218 €$. This device will open the possibility of imaging the esophagus in underprivileged areas.
\end{abstract}

\section{K E Y W O R D S}

fluorescence/autofluorescence imaging, low-cost endoscopy, narrowband imaging, white-light imaging 


\section{1 | INTRODUCTION}

Since their first demonstration in 1806 [1], endoscopes have evolved in several different directions. Endoscopy is an integral part of the healthcare system for the diagnosis of diseases within the esophagus [2], stomach [3], small intestine [4], and large intestine [5]. There are several different types of endoscopes such as white-light [6], narrow-band [7], autofluorescence [8], confocal [9], Raman [10] imaging endoscopes, etc. which are used clinically as diagnostic or research tools. Out of these, white-light, narrow-band, and autofluorescence-based imaging techniques are the most commonly used for the diagnosis of complications related to the gastrointestinal (GI) tract. Although endoscopes have existed for more than two centuries, patients undergoing endoscopic procedures still face the challenge of long waiting times and high costs. Even in developed countries, the wait time ranges from one to three months $[11,12]$, and unfortunately, the situation in underprivileged areas would be even worse. Modern endoscopes contain working channels to take biopsies of the diseased areas for further pathological studies. However, in many cases where the patient shows no sign of complication, biopsies are not required [13]. If the decision on biopsy could be made based on a relatively quick, safe, and more accessible technique in primary care settings, it will reduce the psychological and economic burden on the patients significantly.

Nevertheless, the efforts made in the direction of multimodal low-cost endoscopy without sedation are meager compared to their necessity. Few attempts have been made in the direction of low-cost tethered endoscopes and have been reported for the imaging of the upper GI tract $[14,15]$. Although, such endoscopes are limited to white light imaging capability and have mainly focused on steering capabilities to guide the endoscope through the GI tract, which increases the tether diameter, and therefore patients still need to be anesthetized during the examination.

Yet another technological platform termed as wireless capsule endoscopy $[16,17]$ is well established where a tiny device of the size of a pill can provide images similar to the white light endoscopic images. The pill size device contains a camera, a light source, and once swallowed by the unsedated patients, transmits the images of the GI tract to the acquisition unit wirelessly. Such capsules are again limited to single modality imaging capability and non-reusable thus increasing the cost of the procedure.

The issue of sedation has also been addressed in tethered capsule endoscopy [18], a technological platform to examine the GI tract, where a swallowable capsule is used with a tether diameter of less than $1 \mathrm{~mm}$.
For examination, the patients swallow these capsules like a pill and thus do not require any sedation. Afterward, the capsule is guided through the GI tract by peristalsis without the necessity of any additional guiding mechanism [19]. Such tethered capsules can be easily disinfected for multiuse [29], reducing the effective cost of the procedure significantly. Tethered capsule endoscopes have been developed for optical coherence tomographic imaging [19], tethered confocal capsule microscopy [20], and scanning fiber imaging of the GI tract $[18,21]$ but have not been demonstrated for multimodal imaging.

The focus of previous devices has been specialized imaging techniques and it does not bring the cost and complexity down to a point that it can be easily used in primary care settings. We still lack a low-cost multimodal imaging endoscopic platform that can be used by doctors in primary care settings in low-income areas where access to these facilities is limited. In this direction, in this work, we demonstrated a smartphone-compatible, low-cost, reusable tethered capsule endoscopic platform capable of performing the three commonly used imaging modalities; white-light imaging (WLI), narrow-band imaging (NBI), and fluorescence/autofluorescence imaging (FI/AFI).

\section{2 | MATERIALS AND METHOD}

The picture of the endoscope along with a smartphone is shown in Figure 1A. We used an off the shelf USB powered camera (B083YXRHX7, Ballylelly) working at 30 frames per second and fitted with white light LED's around it for detection and illumination respectively. The camera unit was housed in $10 \mathrm{~mm}$ outer diameter, $6 \mathrm{~mm}$ inner diameter, and $26 \mathrm{~mm}$ long capsule. The imaging window thickness was kept thin $(500 \mu \mathrm{m})$ and the camera with the LED lights was placed in contact with the imaging window to reduce the specular reflections from the outside of the imaging window. The capsule was fabricated in-house from transparent Fluorinated ethylene propylene (FEP), which is known to be a biocompatible material $[20,22]$. The camera processor transferred the images to the USB port using five electrical wires of diameter $0.15 \mathrm{~mm}$ each. These wires were placed in a reinforced polytetrafluoroethylene sheath (OD = $0.95 \mathrm{~mm}$, ID $=0.72 \mathrm{~mm}$, MicroLumen Inc., Oldsmar, Florida). The camera, the video processor, and the LED light source were all operated by a $5 \mathrm{~V}$ power supply delivered from a USB connection. The power emitted from the LED source was approximately $2.8 \mathrm{~mW}$ and an image size of $640 \times 480$ pixels was provided by the camera. The field of view of the camera is around $70^{\circ}$. The 

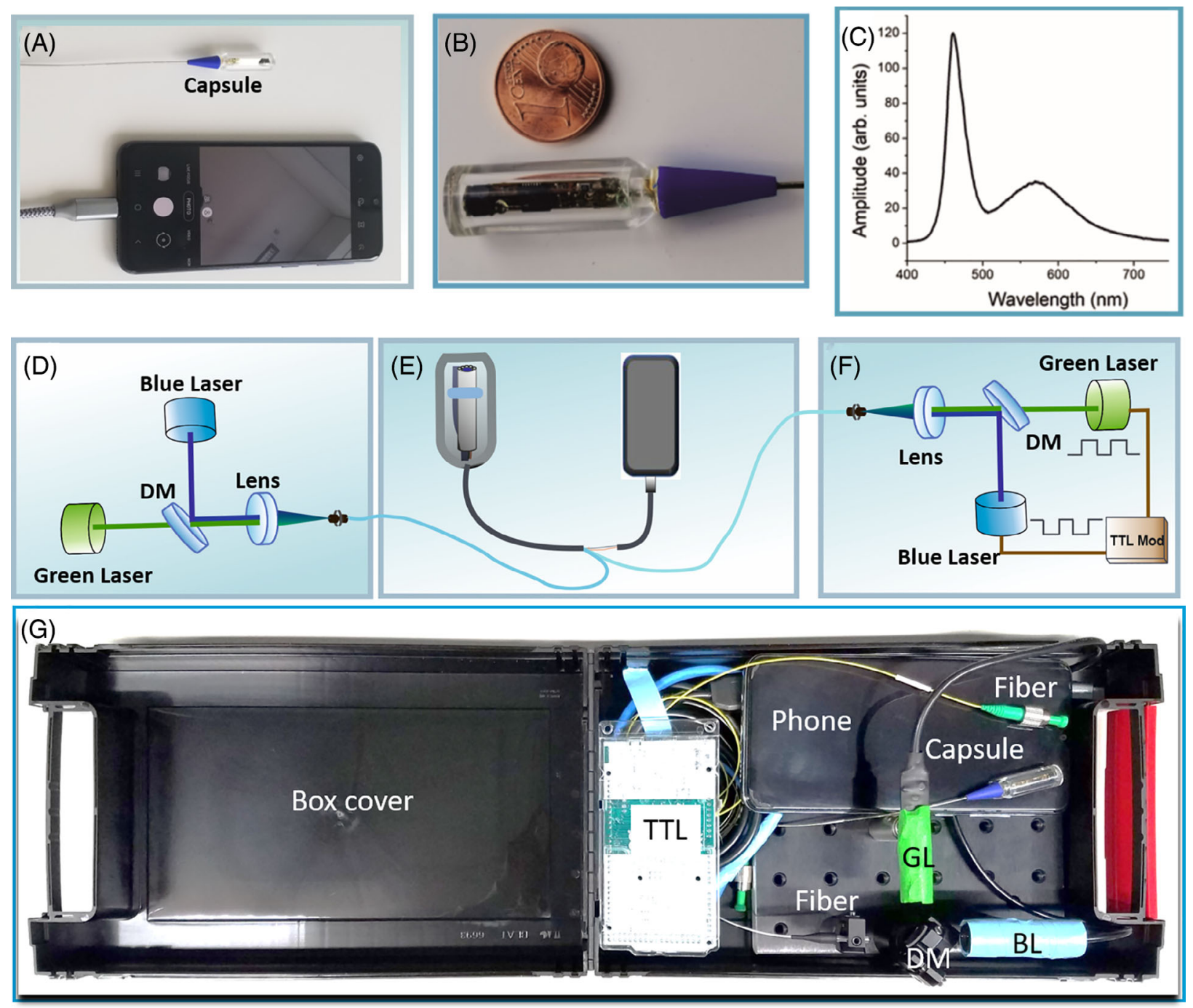

FI G URE 1 A, The picture of the endoscope along with a smartphone. B, Image of the WLI capsule. C, The spectrum of the integrated white light LEDs. D, Schematic of the NBI module where the blue and the green laser light is coupled into a multimode fiber through a lens, and a dichroic mirror (DM). E, Attachable smartphone capsule unit F, Schematic of the FI/AFI module, similar to the NBI, the green, and the blue light is coupled to a multimode fiber through a lens and a dichroic mirror (DM). G, A portable imaging system in a $28 \times 16 \mathrm{~cm}$ box

enlarged picture of the capsule is shown in Figure 1B and the broadband emission spectrum of the white light LED is shown in Figure 1C. This whole unit served as a tethered capsule WLI endoscope. The total weight of the capsule unit was measured to be $3.1 \mathrm{~g}$.

NBI is the method of choice to visualize the vascular system. The absorption spectrum of the hemoglobin has maximum absorption in the blue and the green wavelength ranges. When illuminated with the blue and the green light, blood vessels appear darker compared to the rest of the tissue. Our capsule endoscope provides an option to switch between WLI and NBI by switching between the light sources. To convert the WLI capsule module into an NBI imaging unit, we built an add-on called the laser source module. The schematic of the laser source module is shown in Figure 1D. It consists of a blue
([405 \pm 2$] \mathrm{nm})$ (RLT405-50MG, Roithner Lasertechnik $\mathrm{GmbH}$, Vienna, Austria) and a green diode laser ([532 $\pm 2] \mathrm{nm}$ ) (CW532-050F, Roithner Lasertechnik GmbH, Vienna, Austria). These lasers are easily available at costeffective prices and fortunately even provide intensity stabilities better than $2 \%$. Similar to white light LEDs, these lasers also operate at $5 \mathrm{~V}$. The light from the blue and the green laser was combined into a single beam using a diachronic mirror (DMPL 425 T, Thorlabs Inc., New Jersey) and was coupled to a multimode fiber (core diameter $=200 \mu \mathrm{m}$, outer diameter $240 \mu \mathrm{m}$, FG200LEP, Thorlabs Inc., New Jersey) using a fiber collimator (28938 FS, Germany). The power of the blue and the green laser after the fiber coupling was measured to be 18 and $21 \mathrm{~mW}$ respectively. To convert the WLI capsule into an NBI capsule, a multimode fiber same as the laser 
source module was placed in the sheath along with five electric wires, as shown in Figure 1E. The fiber was connected to the fiber from the laser source module and used as a sample illumination source for the NBI. Speckles were observed at the sample due to the multimode nature of the fiber.

To reduce the effect of speckles, a $50 \mu \mathrm{m}$ thin white plastic was used as a diffuser and was attached in front of the fiber, which helped to provide uniform illumination over the sample. The NBI module is an add-on and the user could switch from NBI to WLI by turning off the NBI module and switching on the white light LEDs.

We also developed a module for fluorescence/ autofluorescence imaging (FI/AFI), as illustrated in Figure $1 \mathrm{~F}$. The WLI module and the NBI module require only a single capsule unit but the FI/AFI requires a separate capsule unit as it needs additional filters in front of the camera. A standard AFI system employs a rotating filter in front of a broadband light source to select a blue and green color for sequential illumination of the tissue. Furthermore, a blue filter is used to block the blue excitation reaching the camera. The autofluorescence light and green reflected light passes through the blue filter giving two sequential images that are combined later. The two images are presented in a single image where the autofluorescence signal is presented in purple color over the green-colored image of the tissue. The whole imaging module could fit in a $28 \times 16 \mathrm{~cm}$ box as shown in Figure 1G.

In our FI/AFI module, we used the same laser source module as the NBI module. To ensure the sequential illumination of the sample with either green or blue light at a time, both the lasers were triggered with a TTL signal at $15 \mathrm{~Hz}$. An ultra-thin green pass filter (35-892, Edmund Optics, USA), which blocks blue light and passes green light, was placed in front of the camera. The fluorescence/ autofluorescence and the green channel images were combined to obtain a single image. Similar to the standard autofluorescence images, the images presented in this work contain fluorescence/autofluorescence information in purple on top of the green-colored widefield image.

To quantify the performance of the designed modules, different tests and studies were performed. All methods carried out in this work are in accordance with relevant guidelines and regulations. For the WLI system, the resolution of the module was determined by imaging the USAF resolution test target (R1L1S1N, Thorlabs Inc., New Jersey) at a distance of $10 \mathrm{~mm}$ from the imaging window. Next, we imaged swine esophagus ex-vivo using the WLI endoscopic capsule system and compared the images with a commercially available white light endoscope (COLOVIEW Mainz, KARL STORZ Endoscopes, Tuttlingen, German). No animal experiments were performed in this study. Only ex-vivo measurements were performed with an esophagus from a dead swine, which was obtained from a local butcher's shop for imaging purposes. To test the potential of the NBI unit, the tongue of a healthy volunteer was imaged sequentially, once with the NBI, and once with the WLI module. Experiments on the lower tongue for narrowband imaging were performed as self-test on the author of the manuscript and no other human experiments were performed in this work.

To demonstrate the capability of the FI/AFI module, we imaged a rectangular piece of a fluorescent paper (VRC1, Thorlabs Inc., New Jersey) placed on top of a non-fluorescent paper. When excited with a blue laser, the fluorescent paper re-emits in a higher wavelength range, whereas standard paper does not provide this signal. The fluorescent paper in our case has a broadband excitation spectrum with a full width of half maximum of approximately $150 \mathrm{~nm}$, centered at $410 \mathrm{~nm}$, and a broadband emission spectrum with a full width of half maximum of approximately $100 \mathrm{~nm}$, centered at $580 \mathrm{~nm}$. We also measured the sensitivity of the FI/AFI module by measuring the fluorescence signals of fluorescein dye with different concentrations. Furthermore, to mimic the autofluorescence imaging in tissue, ex-vivo imaging of swine esophagus was performed after injecting $2.5 \mu \mathrm{M}$ fluorescein dye into the wall of the esophagus. The tissue was excited with $405 \mathrm{~nm}$ blue laser and $532 \mathrm{~nm}$ green laser sequentially and was imaged using our capsule endoscope through a green pass filter. The fluorescence image represented with purple color and green channel image represented with green color was combined to form a composite image similar to standard AFI modules used in clinics.

A breakdown of the cost of the individual components used in the development of the tethered capsule is given in Table 1 and the cost for the components used in the development of the laser source module is given in Table 2.

T A BLE 1 Cost for the capsule components used in different imaging modalities

\begin{tabular}{lccc} 
& WLI $(€)$ & NBI $(€)$ & FI/AFI $(€)$ \\
\hline USB camera & 3.30 & 3.30 & 3.30 \\
Wires & 1.00 & 1.00 & 1.00 \\
\hline Tether & 3.00 & 3.00 & 3.00 \\
Capsule & 4.80 & 4.80 & 4.80 \\
\hline Fiber & - & 2.80 & 2.80 \\
Filter & - & - & 27.50 \\
\hline Total & 12.10 & 14.90 & 42.40 \\
\hline
\end{tabular}


TABLE 2 Cost for the components used in the development of the laser source module which was used in NBI and FI/AFI

\begin{tabular}{lr} 
Green laser & $€ 100.20$ \\
\hline Blue Laser & $€ 23.40$ \\
Dichroic Filter & $€ 55.50$ \\
TTL unit & $€ 28.00$ \\
Fiber Collimator & $€ 10.78$ \\
Total & $€ 217.88$ \\
\hline
\end{tabular}

For all the three imaging modalities, we used an Android-based smartphone (Redmi Note 8 Pro) to record the images of the sample. A freely available image capturing application (Endoscope camera for Android v2.9) was used to view and record the images in the phone memory in MP4 format. The WLI and the NBI can be performed using the smartphone only. Whereas for an AI/AFI module, the image overlapping is required, for which, the recorded images were transferred to a computer for further analysis. The image overlapping for the FI/AFI module was performed using freely available software (ImageJ).

\section{3 | RESULTS}

\section{1 | White light imaging}

WLI module is its main unit. The WLI module contains an off-the-shelf USB powered camera fitted with a white light LED as an illumination source. The power emitted from the white light LED source was approximately
$2.8 \mathrm{~mW}$. The endoscope was designed in a modular manner, where the capsule was designed to perform safe inside body operations. As a first test, the temperature increase in the capsule was measured after running white light LEDs continuously for 10 minutes. The temperature of the capsule wall was monitored using an IR temperature detector. The capsule reached a temperature of $28.5^{\circ} \mathrm{C}$, which is well within the safe limit for inside body operation. Next, the tensile strength of the capsule was tested by attaching a $0.5 \mathrm{~kg}$ weight at its end and hanging it from the top for 10 minutes. This exerts a force of $4.9 \mathrm{~N}$ at its base. Any damage to the assembly was visually inspected and no mechanical damage was found. The capsule was tested against water leakage by submerging it in the water at a depth of $50 \mathrm{~mm}$ for 10 minutes. Water leakage inside the capsule was monitored by visual inspection and no water leakage was observed. The optical performance of the capsule was tested by imaging the USAF resolution target. The resolution target image is shown in Figure 2A. One can see in the image that the first element of the third group is still resolvable with the eye and this group has a line-width of $62.5 \mu \mathrm{m}$. For further justification, a plot of the intensity profile of the line pair is shown in Figure 2B, where the maximum to minimum ratio of the peaks is more than two, which, according to Rayleigh's criterion, are said to be resolved. Therefore, one can claim that the resolution of our WLI endoscope is at least better than $62.5 \mu \mathrm{m}$.

Since the endoscope is developed to provide a platform to examine the upper GI-tract, swine's esophagus was imaged ex-vivo to test its potential. The WLI image of the swine esophagus is shown in Figure 3A. We also imaged the same esophagus using a commercial

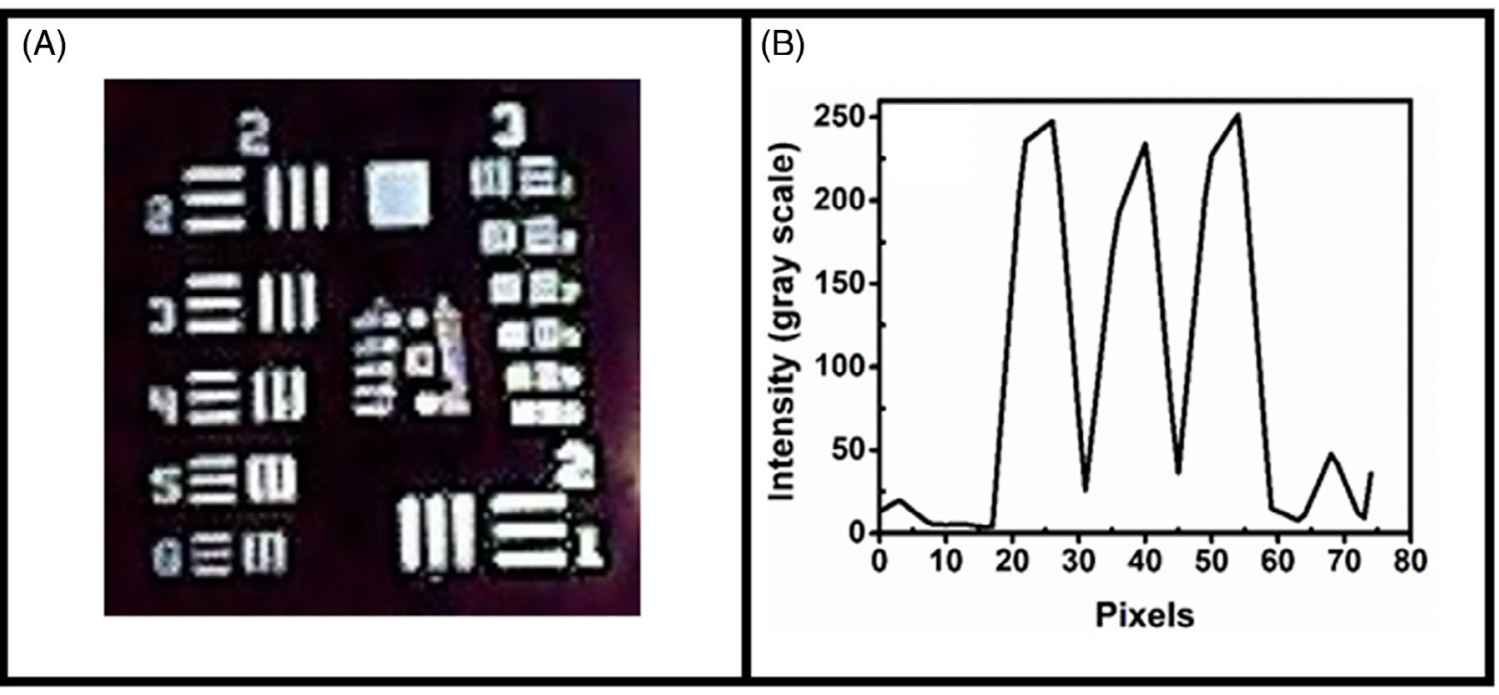

FI G URE 2 A, The image of a resolution target taken with the WLI module. B, Line profile of element 1, group 3 of the resolution target, which corresponds to $62.5 \mu \mathrm{m}$ resolution 


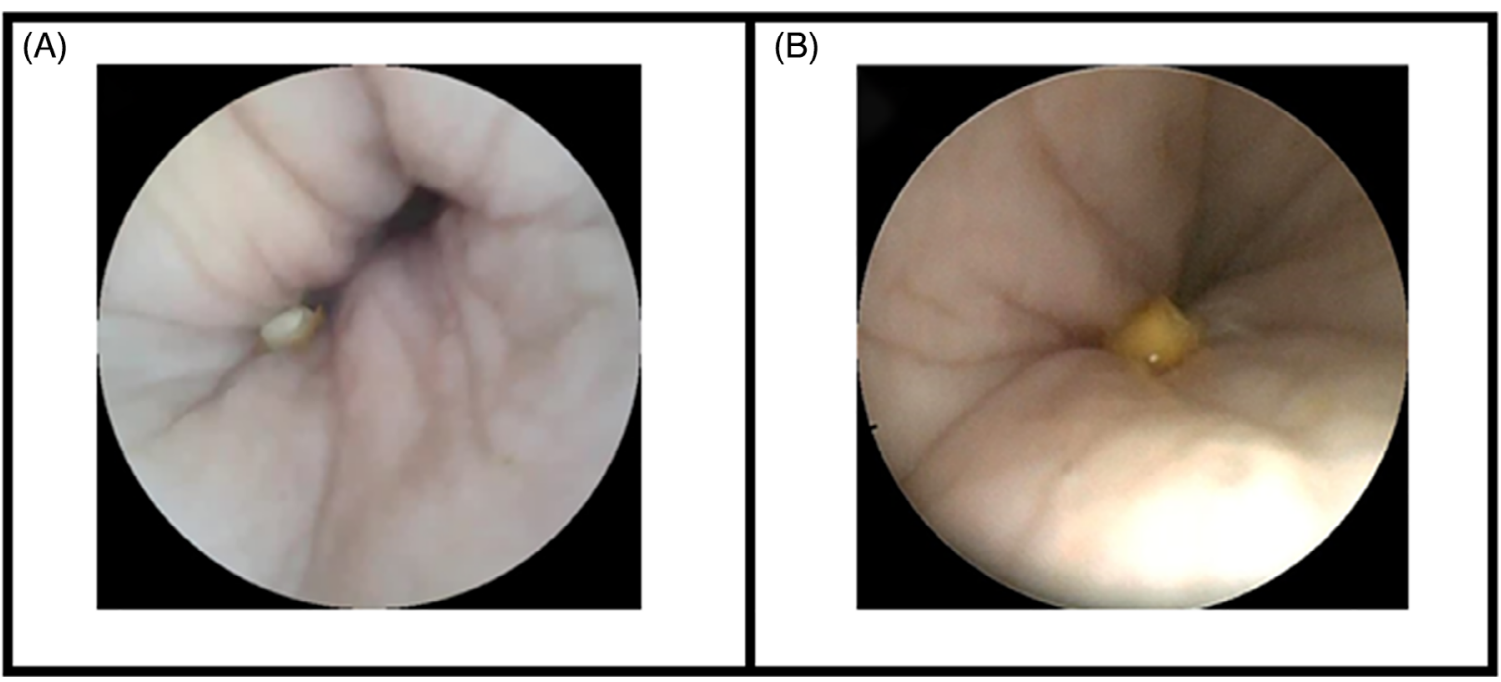

F I G U RE 3 A, The ex-vivo image of the swine esophagus taken with the tethered WLI capsule module. B, The image of the same tissue taken with a commercial endoscope

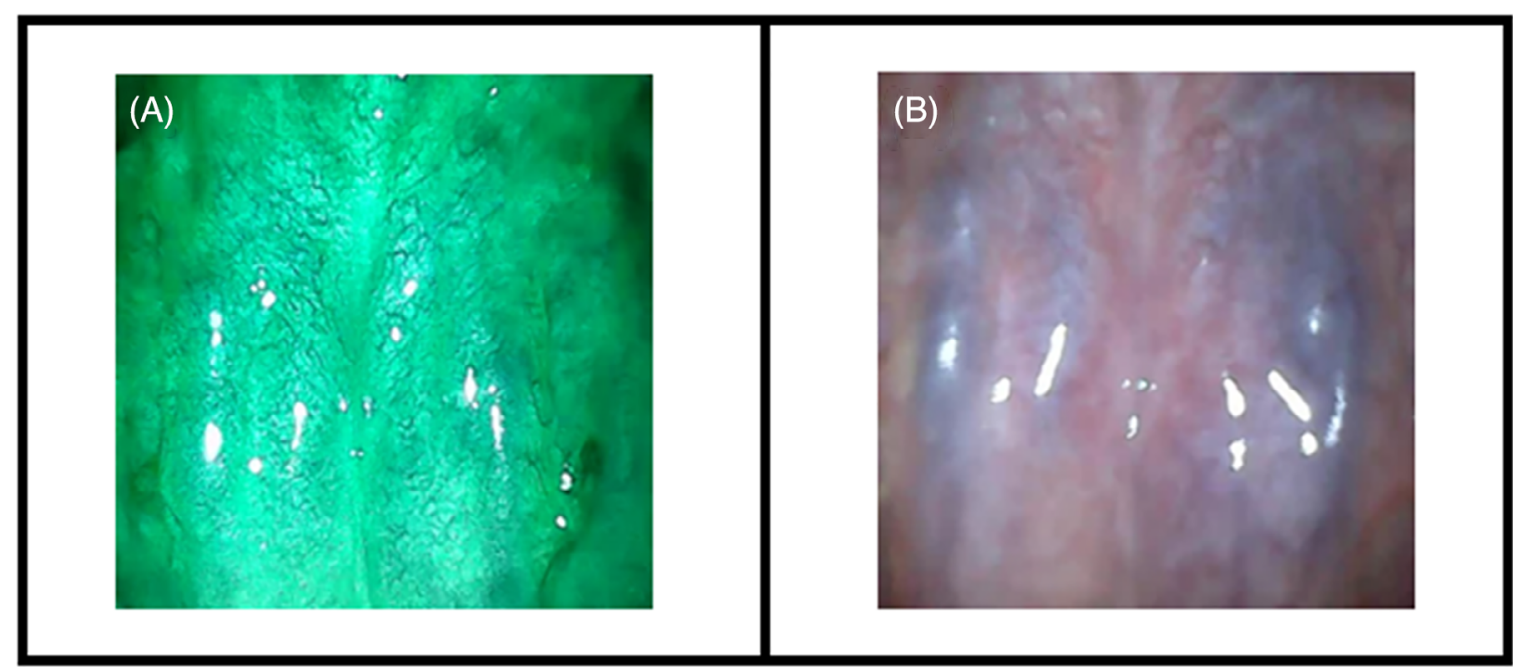

F I G URE 4 A, The image of the lower tongue acquired with the NBI module. B, The lower tongue image acquired with the WLI module

endoscope to compare the performance of our device. The commercial endoscopic image of the esophagus is shown in Figure 3B. One can see that the two images are comparable.

\section{2 | Narrowband imaging}

To test the potential of the NBI module of the capsule endoscope, blood vessels under the tongue of a healthy volunteer were imaged using WLI and NBI mode. The tongue instead of the esophagus was imaged because the tongue provides easy access to the blood vessels whereas the ex-vivo esophagus is usually drained off the blood, which is the main contrast agent for the NBI. The imaging was performed as a self-test which did not require ethical approval. These images are shown in Figure 4, and it is evident that the NBI mode (Figure 4A) provides high contrast images of blood vessels as compared to WLI mode (Figure 4B).

\section{3 | Fluorescence/autofluorescence imaging}

Another module added to our endoscope was FI/AFI. The capability of this module was first tested on a fluorescent paper. On top of a non-fluorescent paper, 
F I G URE 5 A, The image of a fluorescent paper placed on top of a non-fluorescent paper acquired with the WLI module. $B$, The image of the same paper combination acquired with the FI/AFI module. C, The WLI image of the fluorescein dye droplets with different concentrations on a glass slide. D, The image of the same glass slide acquired with the blue laser excitation from the FI/AFI module. E, The graph plotted for the intensity of different dye spots as a function of the fluorescein

concentration. F, The WLI image of the swine esophagus ex-vivo injected with $2.5 \mu \mathrm{M}$ of fluorescein in the wall of the esophagus. G, Image of the same tissue acquired with the FI/AFI module where the fluorescence signal in purple is overlaid the widefield green colored tissue image

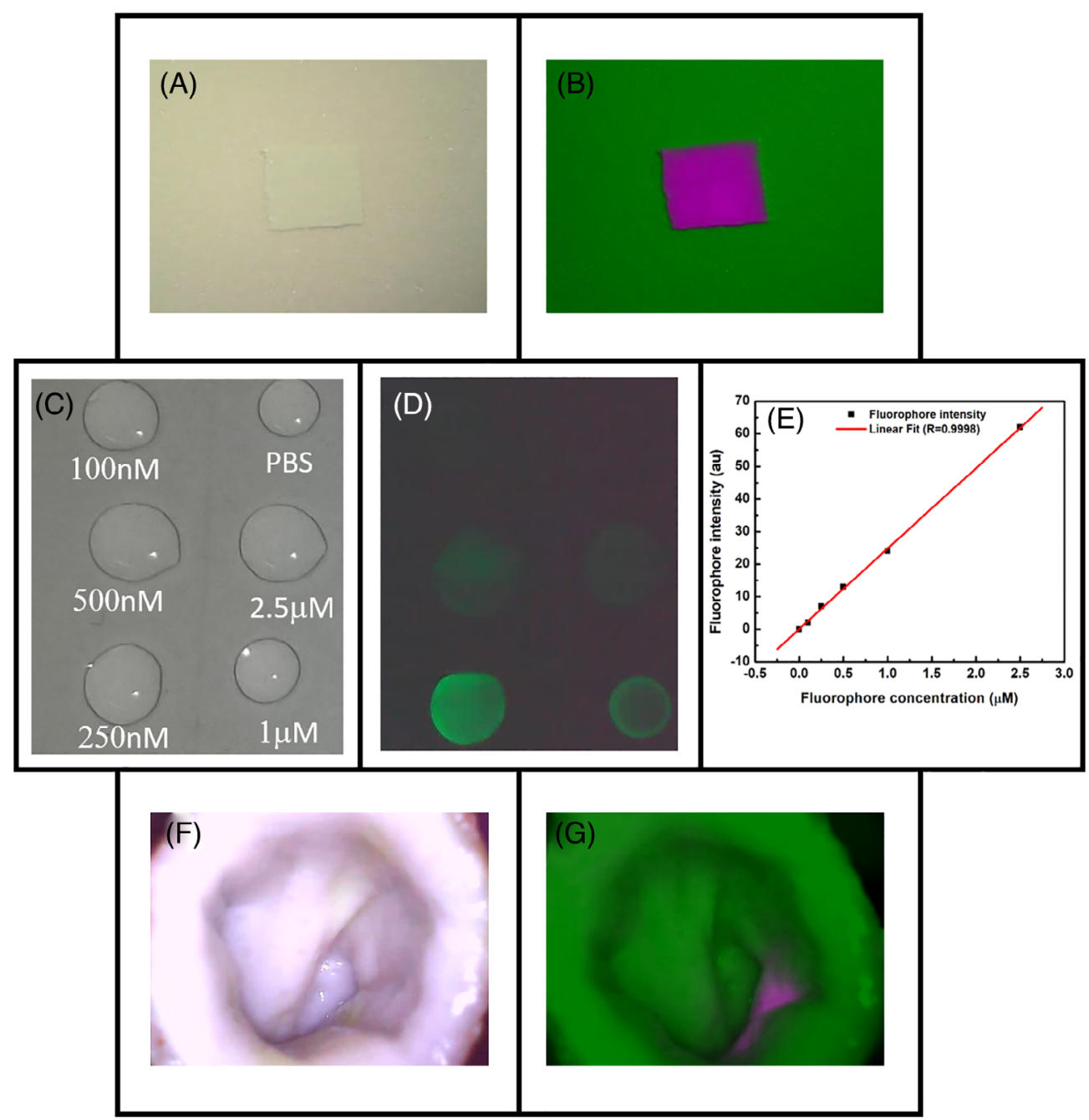

another fluorescent paper was placed. Both these papers were imaged with WLI and FI/AFI modules, as shown in Figure 5A,B respectively. It can be seen clearly that the WLI module shows a minimal difference between the two papers, whereas, in the autofluorescence image, the contrast has been increased manifolds due to the fluorescent signal from the fluorescence paper. To measure the sensitivity of our device, we imaged the fluorescein dye with different concentrations ranging from $100 \mathrm{nM}$ to $2.5 \mu \mathrm{M}$. The white light image of the dye droplets with different concentrations on a glass slide is shown in Figure 5C. Once imaged with the FI/AFI module, (Figure 5D), a graph is plotted between the different concentrations of the dye and their corresponding intensity, and it comes out to be linear (Figure 5E). Further, we injected the swine esophagus wall with $2.5 \mu \mathrm{M}$ fluorescein dye and imaged using the FI/AFI module along with the WLI module of our endoscope. One can see that the image from the WLI unit, shown in Figure 5F, hardly confirms the presence of any fluorophore.
Whereas, the image from the FI module, presented in Figure 5G, contains the signature of the dye in purple color at the injected place on the top of the green-colored tissue image.

\section{4 | DISCUSSION}

We have developed a low-cost smartphone-compatible endoscopic platform for imaging the upper GI tract in low resource settings. The smartphone-compatible endoscopic platform which is based on a low-cost USB camera can be modified to acquire images for one of the three imaging modalities, that is, white-light imaging, narrow-band imaging, or fluorescence/autofluorescence imaging. Whereas the WLI module and the NBI module can use the same capsule, FI/AFI module requires a different capsule as it requires filters that are not compatible with the WLI and the NBI. The reader should be aware that the three kinds of imaging cannot be 
performed simultaneously and requires either changing the capsule unit such as between WLI/NBI and FI/AFI or switching between the light sources when changing from the WLI to the NBI. The white-light endoscopic capsule costs approximately $12 €$, whereas the NBI and the FI/AFI capsules cost approximately $15 €$ and $42 €$ respectively. In addition to the capsule, the NBI, and the FI/AFI require additional lasers for the tissue illumination. The cost of the laser source module is approximately $218 €$. Our WLI module design cost is lower than the other reported low-cost endoscopes in the literature (USD 35) [14]. Furthermore, for the previously reported endoscope, the capsule and the tether diameter is 13.5 and $7 \mathrm{~mm}$ respectively [14], compared to the capsule and the tether diameter used in our design which is 10 and $0.95 \mathrm{~mm}$ respectively, making endoscopy possible by swallowing the capsule instead of sedating the patients. Other earlier reported low-cost endoscopes are either using expensive cameras [15], or scanning mechanism [18], which increases their cost significantly. Like other capsule endoscopes $[14,15,18,19]$, our device is also reusable as it can be disinfected easily, as reported in earlier works $[29,20,23]$. Thus, in reality, the cost for each examination is lowered further. The most expensive parts of the system, the laser source module for the NBI, and the FI/AFI is detachable. Therefore, it is possible to use them with multiple tethered capsule endoscopic units over a long time. The camera used in our endoscope has $640 \times 480$ pixels, although it is not a high definition camera compared to the previous works [14], with developing technology, better cameras are becoming readily available with similar cost, and can be used for future work. The field of view of our endoscope is $70^{\circ}$, which is a little less compared to the other reported work (around $100^{\circ}$ ) $[15,18]$, but it can be further improved by using different optics combination in front of the camera. Nevertheless, all the previously reported $[14,15,18$, 21] tethered capsules are designed for single modality imaging only, whereas our approach offers NBI and FI/AFI module along with WLI, which is a significant advancement in the field of capsule endoscopy. The detection sensitivity of our endoscope is approximately $100 \mathrm{nM}$ for fluorescein dye. Previous works have reported sensitivity of $5 \mathrm{nM}$ for scanning fiber capsule endoscope [21] using fluorescein dye and $12.5 \mu \mathrm{M}$ for wireless-capsule using flavin adenine dinucleotide [17]. The detection sensitivity of our system can be further increased by increasing the excitation laser power or increasing the camera integration time. The whole system can fit in a $(28 \times 16 \mathrm{~cm})$ box, making it extremely portable.

Commercial endoscopes have in general twice the resolution than our reported endoscope at the same working distance. If required, the resolution of our endoscope can be improved further by adding a tightly focusing lens in front of the camera assembly. The WLI module was tested for its biomedical applicability by imaging swine esophagus ex-vivo and image quality was found to be comparable with the images acquired using a commercial endoscope, suggesting the suitability of our endoscope for the upper GI imaging.

Imaging vascular structures within the esophagus also provide critical information for a complete diagnosis of the disease. In our endoscopic capsule, the vascular information was obtained by adding the NBI module. The increased visibility of the vascular structures makes our endoscope capable of imaging changes in vascularization due to any anomaly. Along with this, the FI/AFI unit is added to this device to obtain fluorescence information of the tissue which opens up the possibility for real-time fluorescence/autofluorescence imaging using this low-cost device.

No steering capability is one of the limitations of our design. However, such a capability is not required for esophageal imaging as the capsule is guided by peristalsis within the esophagus $[18,19,20,24,25]$. A major concern for such a design could be patient safety, as there is a possibility that during the procedure there is detachment of the capsule. However, our capsule design follows the previously reported tethered capsule design $[18,19,24,25]$, which has been used clinically in hundreds of patients [19, 20, 23, 29]. The capsule endoscope has been designed with biomedical grade material and can be tested for tensile strength, water, or chemical leaks before administering to the patients to minimize the potential hazards. The reader should be aware that the developed capsule should be compatible with the low $\mathrm{pH}$ conditions inside the stomach. We did not perform an acid test to verify the compatibility of the developed capsule for low $\mathrm{pH}$ conditions. However, we fabricated the capsule using the same biocompatible material which has been used previously to develop capsules suitable for the stomach imaging under low pH (1) conditions [29]. Similar capsule designs have already been approved for clinical trials in the United States under the category of capsule endoscopy of the esophagus for patients [26, 27] with chronic reflux, and those with a known history of Barrett's esophagus. No case of capsule detachment has been reported yet for tethered capsule endoscopy but in the unlikely case of detachment, the capsule is expected to pass through the GI tract.

One important consideration while using tethered systems is the pullback speed while imaging long tubular structures such as the esophagus. The two factors which need consideration are the patient's comfort and the operator's ability. Generally, patients would be more 
comfortable with slower pullback speed but from the operator's point of view, it is challenging to perform the pullback at slower speeds. In previous studies [29], it was found that the average pullback speed was approximately $30 \mathrm{~mm}$ per second when manual pullback was performed. When the data is acquired while the tethered capsule is moving, image blurring will occur. For our capsule, the image blurring was calculated to be approximately $6 \%, 15 \%$, and $49 \%$ when the capsule was pulled back with the speed of 7,15 , and $30 \mathrm{~mm} / \mathrm{s}$ respectively, based on the previously proposed algorithms [28]. Since the blurring in the image acquisition increases with the increased pullback speed, it is advisable to use a motorized pullback mechanism that can pull at slower speeds compared to the clinical operator.

It should be noted that we used a fixed diffuser in front of the laser delivery fiber in the designed capsule to minimize the effects of speckles in the sample illumination. Whereas a moving diffuser can effectively reduce the speckles in the illumination, a fixed diffuser is only effective if the speckles produced on the sample are much smaller than the resolution of the imaging system [30]. For the designed capsule, we measured the average speckle size at a distance of $10 \mathrm{~mm}$ from the imaging window and was found to be approximately $8 \mu \mathrm{m}$. Since the lateral resolution of the system was approximately $62.5 \mu \mathrm{m}$ which is about 8 times larger than the speckle size in the illumination, we can safely say that the illumination was homogenous. The same is evident from the images in the results section where the effect of speckles is hardly noticeable. In the future, we can also try to include a piezo actuator over the fiber to reduce the speckles if deemed necessary.

\section{5 | CONCLUSION}

In conclusion, we have designed, developed, and tested a smartphone-compatible, low-cost, reusable, tethered capsule endoscopic platform that is capable of whitelight, narrowband, and fluorescence/autofluorescence imaging. Different modules were tested for their performance. The resolution of the WLI unit was measured to be better than $62.5 \mu \mathrm{m}$. A normal swine esophagus was imaged to compare the capabilities of the WLI module to the commercial endoscope. The effectiveness of the NBI module was demonstrated by imaging the tongue of a healthy volunteer. FI/AFI modules capabilities was first demonstrated by imaging a fluorescence and a non-fluorescence paper, then its sensitivity was measured using fluorescein dye, and finally, to test its real-time applications, a fluorescein dye injected swine esophagus was imaged ex-vivo using FI and WLI modules sequentially. With all the features of WLI, NBI, and FI/AFI added together along with its overall low cost, we believe that this tethered capsule endoscope will aid the clinicians in diagnosing esophagus-related disorders in low resource settings.

\section{ACKNOWLEDGMENTS}

GS would like to thank Dr. Jochen Guck for funding support. Open access funding enabled and organized by Projekt DEAL.

\section{AUTHOR CONTRIBUTIONS}

Kanwarpal Singh, Gargi Sharma, and Maximilian Waldner designed the study. Oana-Maria Thoma, Gargi Sharma, Kanwarpal Singh, Katharina Blessing acquired data during studies. Gargi Sharma, Kanwarpal Singh, Maximilian Waldner contributed to data interpretation. Gargi Sharma and Katharina Blessing wrote the manuscript with key input from all other co-authors. Kanwarpal Singh is the principal investigator and provided funding for the study.

\section{CONFLICTS OF INTEREST}

The authors declare no potential conflict of interest.

\section{DATA AVAILABILITY STATEMENT}

The datasets generated and analyzed in the article are available from the corresponding author upon reasonable request.

\section{ORCID}

Gargi Sharma (10) https://orcid.org/0000-0002-8666-7820 Katharina Blessing (1) https://orcid.org/0000-0002-23953440

Maximilian Waldner (10) https://orcid.org/0000-0002-55607665

\section{REFERENCES}

[1] P. Bozzini, J. Practischen Arzneykunde Wundarzneykunst (in German) 1806, 24, 107.

[2] J. M. Streitz, C. W. Andrews, F. H. Ellis, J. Thorac. Cardiovasc. Surg. 1993, 105, 383.

[3] I. Oda, H. Kondo, T. Yamao, D. Saito, H. Ono, T. Gotoda, H. Yamaguchi, S. Yoshida, T. Shimoda, Endoscopy 2001, 33, 507.

[4] H. Yamamoto, H. Kita, K. Sunada, Y. Hayashi, H. Sato, T. Yano, M. Iwamoto, Y. Sekine, T. Miyata, A. Kuno, H. Ajibe, K. Ido, K. Sugano, Clin. Gastroenterol. Hepatol. 2004, 2, 1010.

[5] D. A. Redelmeier, J. Katz, D. Kahneman, Pain 2003, 104, 187.

[6] M. Kriegmair, D. Zaak, K.-H. Rothenberger, J. Rassweiler, D. Jocham, F. Eisenberger, R. Tauber, A. Stenzl, A. Hofstetter, J. Urol. 2002, 168, 475.

[7] N. Horiguchi, T. Tahara, T. Kawamura, M. Okubo, S. Tahara, M. Nagasaka, Y. Nakagawa, T. Shibata, N. Ohmiya, J. Gastrointest. Liver Dis.: JGLD. 2017, 26, 357. 
[8] J. Haringsma, G. N. J. Tytgat, H. Yano, H. Iishi, M. Tatsuta, T. Ogihara, H. Watanabe, N. Sato, N. Marcon, B. C. Wilson, R. W. Cline, Gastrointest. Endosc. 2001, 53, 642.

[9] G. E. Tontini, J. Mudter, M. Vieth, R. Atreya, C. Günther, Y. Zopf, D. Wildner, R. Kiesslich, M. Vecchi, M. F. Neurath, H. Neumann, Endoscopy 2015, 47, 437.

[10] O. Chernavskaia, S. Heuke, M. Vieth, O. Friedrich, S. Schürmann, R. Atreya, A. Stallmach, M. F. Neurath, M. Waldner, I. Petersen, M. Schmitt, T. Bocklitz, J. Popp, Sci. Rep. 2016, 6, 29239.

[11] M. Lungen, B. Stollenwerk, P. Messner, K. W. Lauterbach, A. Gerber, Int. J. Equity Health 2008, 7, 1.

[12] R. M. Janssen, O. Takach, E. Nap Hill, R. A. E. Can, J. Gastroenterol. Hepatol. 2015, 2016, 11.

[13] A. Peixoto, M. Silva, P. Pereira, G. Macedo, GE Port. J. Gastroenterol. 2015, 23, 19.

[14] N. Garbin, A. P. Mamunes, D. Sohn, R. W. Hawkins, P. Valdastri, K. L. Obstein, Endosc. Int. Open 2019, 07, E1175E1183.

[15] A. Z. Taddese, P. R. Slawinski, K. L. Obstein, P. Valdastri, Rob. Sci. Syst.: Online Proc. 2016, 2016, 1139.

[16] R. Eliakim, Z. Fireman, I. M. Gralnek, K. Yassin, M. Waterman, Y. Kopelman, J. Lachter, B. Koslowsky, S. N. Adler, Endoscopy 2006, 38, 963.

[17] M. A. Al-Rawhani, J. Beeley, D. R. S. Cumming, Sci. Rep. 2015, 5, 18591.

[18] E. J. Seibel, R. E. Carroll, J. A. Dominitz, R. S. Johnston, C. D. Melville, C. M. Lee, S. M. Seitz, M. B. Kimmey, IEEE Trans. Biomed. Eng. 2008, 55, 1032.

[19] M. J. Gora, J. S. Sauk, R. W. Carruth, K. A. Gallagher, M. J. Suter, N. S. Nishioka, L. E. Kava, M. Rosenberg, B. E. Bouma, G. J. Tearney, Nat. Med. 2013, 19, 238.

[20] N. Tabatabaei, D. Kang, M. Kim, T. Wu, C. N. Grant, M. Rosenberg, N. S. Nishioka, P. E. Hesterberg, J. Garber, Q. Yuan, A. J. Katz, G. J. Tearney, Sci. Rep. 2018, 8, 2631.

[21] C. Yang, V. W. Hou, E. J. Girard, L. Y. Nelson, E. J. Seibel, J. Biomed. Opt. 2014, 19, 076014.
[22] C. A. Homsy, K. D. Ansevin, W. O'Bannon, S. A. Thompson, R. Hodge, M. E. Estrella, J. Macromol. Sci.: Part A - Chem. 1970, 4, 615.

[23] H. C. Wolfsen, P. Sharma, M. B. Wallace, C. Leggett, G. Tearney, K. K. Wang, Gastrointest. Endosc. 2015, 82, 631.

[24] M. J. Gora, L. Quénéhervé, R. W. Carruth, W. Lu, M. Rosenberg, J. S. Sauk, A. Fasano, G. Y. Lauwers, N. S. Nishioka, G. J. Tearney, Gastrointest. Endosc. 2018, 88, 830-840.e833.

[25] M. Gora, L. Simmons, L. Quénéhervé, C. Grant, R. Carruth, W. Lu, A. Tiernan, J. Dong, B. Walker Corkery, A. Soomro, M. Rosenberg, J. Metlay, G. Tearney, J. Biomed. Opt. 2016, 21, 104001.

[26] U. o. Washington in Tethered Capsule Endoscope in Screening Patients With Barrett Esophagus, Vol. (Ed.^Eds.: Editor), City, 2016, pp. NCT02729948.

[27] M. G. Hospital in Coordinating Center: Natural History of Barrett's Esophagus Using Tethered Capsule Endomicroscopy, Vol. (Ed.^Eds.: Editor), City, 2018, pp. NCT03459339.

[28] M. Elsayed, F. Sammani, A. Hamdi, A. Al-Baser, H. Babalghoom, Int. J. Simul.: Syst. Sci. Technol. 2019, 19, 4.

[29] M. J. Gora, L. H. Simmons, L. Quénéhervé, C. N. Grant, R. W. Carruth, W. Lu, A. Tiernan, J. Dong, B. Walker-Corkery, A. Soomro, M. Rosenberg, J. P. Metlay, G. J. Tearney, J. Biomed. Opt. 2016, 21, 104001.

[30] B. Kondász, B. Hopp, T. Smausz, J. Eur. Opt. Soc.-Rapid Publ. 2018, 14, 27.

How to cite this article: Sharma G, Thoma O-M, Blessing K, Gal R, Waldner M, Singh K. Smartphone-based multimodal tethered capsule endoscopic platform for white-light, narrow-band, and fluorescence/autofluorescence imaging. $J$. Biophotonics. 2021;14:e202000324. https://doi.org/ 10.1002/jbio. 202000324 